\title{
Miniaturized Implantable DS-PIFA Antenna for Biomedical Applications
}

\author{
Farhad Gozasht \\ Centre for Health Technologies \\ School of Electrical, Mechanical and \\ Mechatronic Systems \\ Faculty of Engineering and IT, \\ University of Technology Sydney, 2007, Australia \\ Farhad.gozasht@uts.edu.au
}

\author{
Ananda Sanagavarapu Mohan \\ Centre for Health Technologies \\ School of Electrical, Mechanical and \\ Mechatronic Systems \\ Faculty of Engineering and IT, \\ University of Technology Sydney, 2007, Australia \\ Ananda.sanagavarapu@uts.edu.au
}

\begin{abstract}
This paper presents an implantable miniaturized planar inverted-F antenna (PIFA) for biomedical applications. We propose small sized PIFA that can operate over $2.45 \mathrm{GHz}$ Industrial, Scientific, and Medical (ISM) band. We have investigated the effect of antenna parameters on resonant frequencies using empirical parametric studies and achieved highly compact PIFA antenna occupies a volume of $92 \mathrm{~mm}^{3}$ with a size of $8.1 \times 7.1 \times 1.6 \mathrm{~mm}^{3}$. Moreover, the effects of the variation of surrounding environment such as skin and fat thickness on the antenna performance are also considered. The impedance characteristics of the proposed antennas are measured using experimental tissue mimicking phantoms. We have also evaluated the calculated Specific Absorption Rate (SAR) by using an indirect thermal method to measure the SAR inside the mimicking phantom.
\end{abstract}

\section{Categories and Subject Descriptors}

B.4.1 - Data Communication Devices.

\section{General Terms}

Design, Experimentation, Measurement and Verification.

\section{Keywords}

Biomedical implantable antennas, Wireless Medical Telemetry, Electrically small antennas, SAR.

\section{INTRODUCTION}

Major challenges faced by the design of antennas for implantation inside human body include miniaturization, improved radiation performance and bandwidth enhancement [1-3]. The radiation efficiency of an implantable antenna decreases due to the high dielectric loss caused by the surrounding human tissues. The smaller size and lower efficiencies coupled with low power constraints can lead to a shorter communication range. To overcome these challenges, many improved implantable antenna designs have been proposed in the literature [2], [4]. Folded dipoles have simpler structure but their radiation efficiency is $0.14 \%$ which is quite low [2]. To improve this, a multi-layered spiral antenna was proposed in [4] with a radiation efficiency about $0.5 \%$ at $2.45 \mathrm{GHz}$, which is still low. An implantable, miniaturized annular slot antenna having size of $22 \times 20 \mathrm{~mm}^{2}$ was reported in [3] with a radiation efficiency of $1.2 \%$ which is an improvement over existing works [2], [4]. It is also important to consider trade-off between antenna size and achievable radiation efficiency. In addition, impedance bandwidth is another important parameter for body-implanted antennas as larger bandwidth can accommodate possible shifts in the resonant frequency due to variation in human tissue properties or other perturbations that may occur during implantation. To satisfy these, a cavity loaded patch antenna was reported in [5], and yet, the best antenna size achieved was only $10 \times 10 \times 1.27 \mathrm{~mm}^{3}$ at $2.45 \mathrm{GHz}$ with a $7.7 \%$ impedance bandwidth.

In order to obtain improved performance, this paper proposes a miniaturized implantable Dual-Slot (DS) PIFA antenna which operates over ISM band at $2.45 \mathrm{GHz}$, and occupies a volume of only $92 \mathrm{~mm}^{3}$ with a compact size of $8.1 \times 7.1 \times 1.6 \mathrm{~mm}^{3}$. The antenna has a measured bandwidth of $330 \mathrm{MHz}(13.5 \%)$ and provides a reasonably moderate radiation efficiency of $2.5 \%$ when immersed inside a human-tissue mimicking numerical phantom which is better than the reported results in the literature [2-5]. We also report measured SAR performance when the proposed antenna is implanted inside an experimental tissue mimicking phantom. The paper is organized as follows: in section 2, we present the antenna design procedure, and section 3 provides information about the human tissue mimicking phantoms. In section 4, measurements are reported and the conclusions are provided in section 5 .

\section{DESIGN PROCEDURE}

The geometry of the proposed miniaturized implantable DS-PIFA printed on a FR-4 substrate is shown in figure 1. The antenna design procedure has been carried out in two steps: first, the dimensions of the dual slot antenna having a shorting pin and feed were obtained using empirical formulas [6] to operate at 2.45 $\mathrm{GHz}$ in air. Then, using $\mathrm{FEKO}^{\mathrm{TM}}$ [7], the antenna model was immersed inside a homogeneous tissue mimicking numerical phantom to obtain its geometrical dimensions for operation in the $2.45 \mathrm{GHz}$ band. Next, a thin dielectric superstrate was added to the antenna and the FEKO calculations were carried out when it is embedded inside a multi-layered human tissue mimicking numerical phantom mainly to fine tune the geometrical 


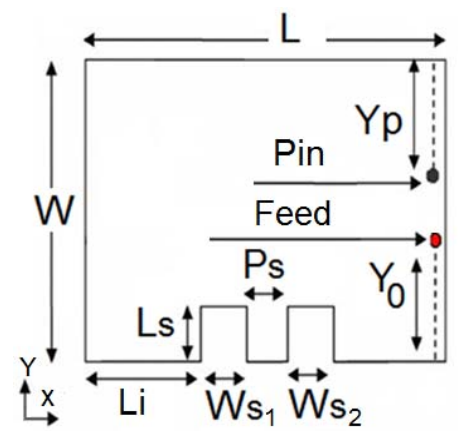

Figure 1: Geometry of proposed PIFA.

dimensions of the antenna to obtain resonance in the $2.45 \mathrm{GHz}$ band. Detailed parametric studies have been conducted in order to quantify the effects of the position of shorting pin, feed point as well as different tissue parameters of the phantom to empirically optimize the antenna geometry. Referring to figure1, the dimensions of the rectangular-shaped patch as well the two radiating slots are the key parameters for antenna operation. In addition, the locations of the shorting pin and the coaxial feed are also important to obtain good matching within the chosen band. The advantage of the DS-PIFA geometry is that it could provide significantly larger bandwidths than conventional patch antennas as its structure helps to obtain antenna resonance at two close resonant frequencies thus leading to the creation of wideband operation. The calculated results on reflection coefficient showed that a ground plane that is slightly larger than the metallic patch can obtain the reflection coefficient better than $-10 \mathrm{~dB}$ within the band of interest. It has been found that the antenna can perform optimally when the feed and the shorting pin are placed on the symmetric central vertical axis with respect to the slots. The geometrical dimensions of the DS-PIFA were $19 \times 17 \times 1.6 \mathrm{~mm}$ to operate at $2.45 \mathrm{GHz}$ inside a lossy human tissue mimicking phantom. The antenna has a simple coaxial feeding structure. The dielectric permittivity and conductivity of the FR4 substrate are 4.4 and 0.025 respectively. The metallic patch has been covered by a superstrate having the same dielectric constant and thickness as that of the substrate.

We have conducted parametric studies to investigate the effects of each geometrical parameter of the proposed antenna on the resonant frequency and impedance bandwidth with an aim to further miniaturize the antenna. These investigations included investigating the effects of the variation of the positions of feed and shorting pin, the variation of widths and lengths of slots plus their symmetry. In each of these cases only one of the parameters was varied while keeping other antenna parameters fixed. It was found that the slot width variation contributes to variation of the antenna resonant frequency. Our investigations also highlighted that for certain feed and pin positions on the patch, the slot lengths could be reduced without affecting the resonance at 2.45 $\mathrm{GHz}$. When the slot length was reduced, the area of the patch increased making the antenna to resonate at lower frequencies. By appropriately reducing the patch area and varying the pin position, we could obtain further size reduction of the antenna without affecting the resonant frequency at $2.45 \mathrm{GHz}$ band. The initial size of $19 \times 17 \times 1.6 \mathrm{~mm}$ has now been reduced to $8.1 \times 7.1 \times 1.6 \mathrm{~mm}$, i.e., a $58 \%$ reduction in the antenna physical size is achieved still operating at $2.45 \mathrm{GHz}$. The resultant miniaturized PIFA is quite small compared to other reported implantable antennas in the literature [2-5].

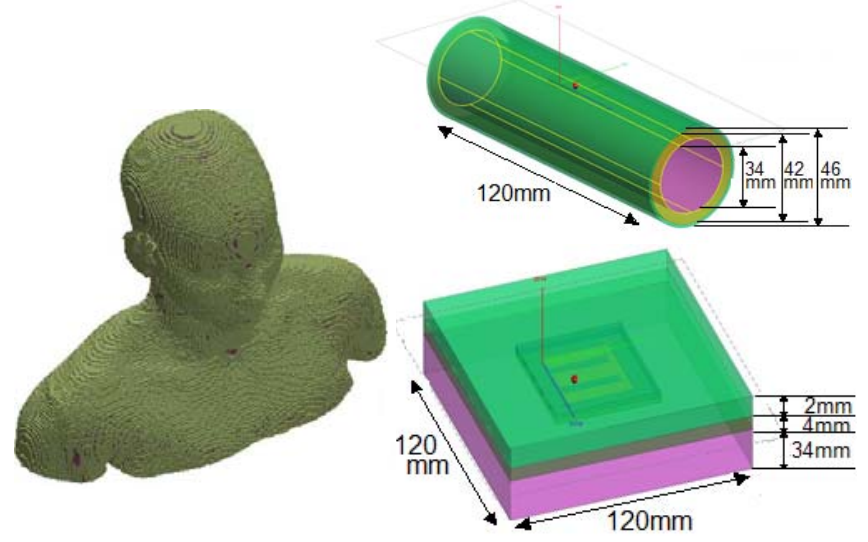

Figure 2: Multi-layered human tissue numerical phantoms. a) Complex human body phantom, b) Layered cylindrical and Layered Block phantoms.

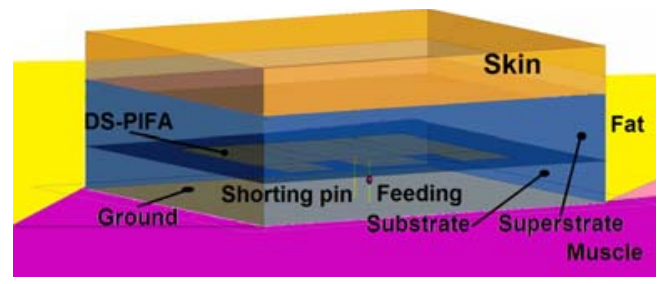

Figure 3: 3D view in Numerical Multilayered Tissue Mimicking phantom.

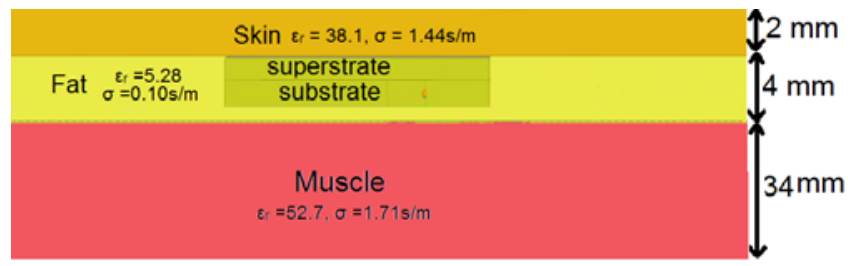

Figure 4. Side view in mimicking phantom.

\section{TISSUE MIMICKING PHANTOMS}

\subsection{Numerical Phantoms}

The numerical phantoms used in simulations are shown in figure 2 in which the DS-PIFA is immersed inside inhomogeneous multi layered complex human body model. Figure 3 and 4 show the position of the proposed implantable PIFA inside the numerical multilayered phantoms. The antenna is embedded into the fat layer under the skin and above the chest muscles. The dielectric properties [10] and the thickness of each tissue layer are shown in figure 4 . The complex human body phantom provides accurate results; however, it requires a lot of computational resources for each run. So, we used alternate, smaller, multi-layered blockshaped and cylindrical shaped numerical phantoms but using the same dielectric properties for skin, fat and muscle layers as shown in figure 4(b). It is expected that the cylindrically shaped multilayered phantom can simulate antenna implantation inside the arm whereas the layered rectangular block phantom simulates antenna implantation under the chest skin. Results obtained using FEKO on reflection coefficient and radiation efficiency using these smaller, but layered numerical phantoms demonstrate a good agreement with those obtained using complex human body numerical phantom as shown in figure 5 . 


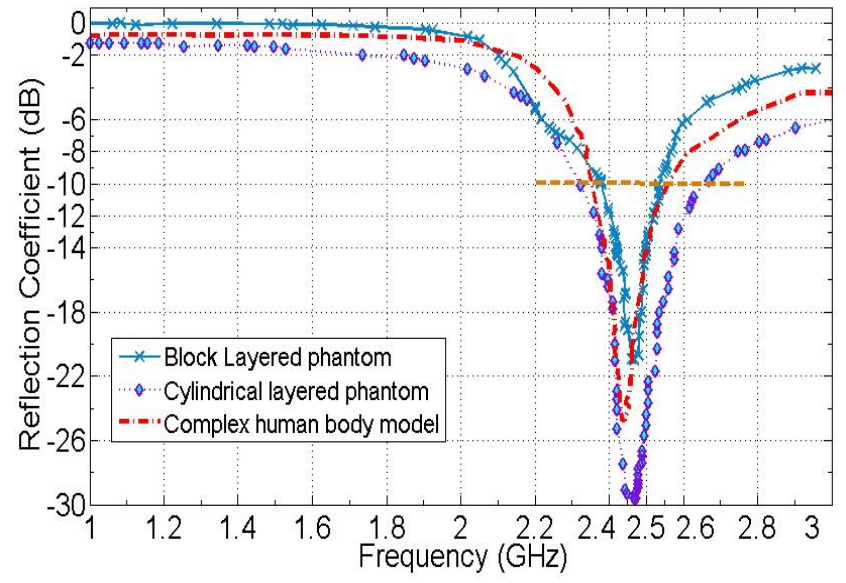

Figure 5: Calculated Reflection coefficient of Dual-slot PIFA: Layered Block and cylindrical phantoms versus Complex human body phantom.

The calculated radiation efficiency of the proposed antenna including the superstrate layer is $2.5 \%$ when immersed inside a complex human body model. As expected, the calculated efficiency is low due to the presence of lossy tissues. However, the efficiency is much better than those published for similar class of antennas in the literature [2-5].

We have also included in our simulations, other factors that may affect the antenna performance during implantation and testing in realistic scenarios. These include variations in dielectric properties and thickness of the fat and skin layers of experimental vivo models. The proposed PIFA performs well in simulations even with reasonable changes in tissue dielectric parameters and thicknesses so that the coverage of $2.45 \mathrm{GHz}$ band can be guaranteed for a variety of human tissue parameters.

\subsection{Experimental Mixed-Meat Phantom}

To verify the numerical performance, we have conducted measurements inside an experimental phantom whose dielectric properties are close to the tissue properties. For this, we developed a mixed-meat phantom that is prepared with a mixture of lean pork and beef. The conductivity and dielectric constants of the mixture were measured by using dielectric probe kit and Agilent E5071C VNA from $100 \mathrm{MHz}$ to $3 \mathrm{GHz}$ by placing the mixture in a plastic container of size $120 \times 120 \times 45 \mathrm{~mm}^{3}$. A good agreement with the desired dielectric properties was observed and comparison of relative permittivity and conductivities of the mixed meat, skin, and muscle are shown in figure 6 .

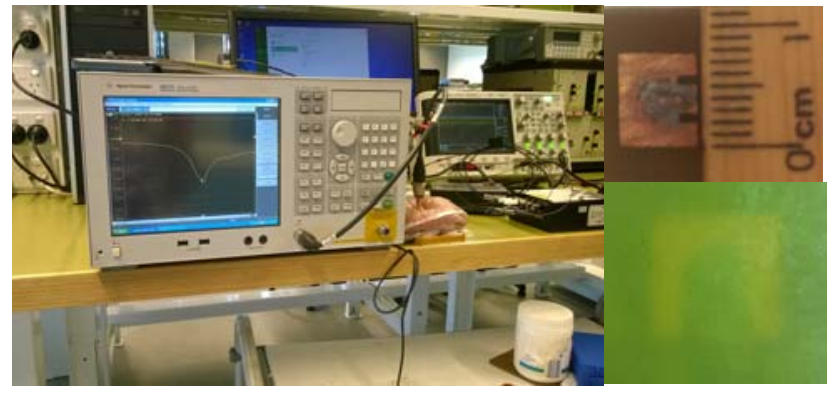

Figure 7. Measurement setup and proposed PIFA on FR4 substrate.

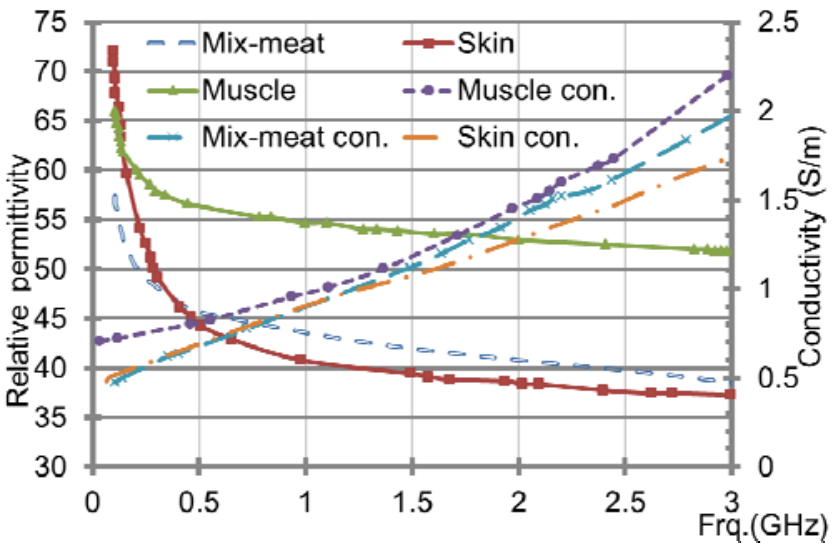

Figure 6: Comparison of relative permittivity and conductivity of the mixed-meat phantom with skin, and muscle.

It can be observed that the relative permittivity of mixed meat phantom is closer to human skin which is useful as the antenna is placed very close to the skin layer.

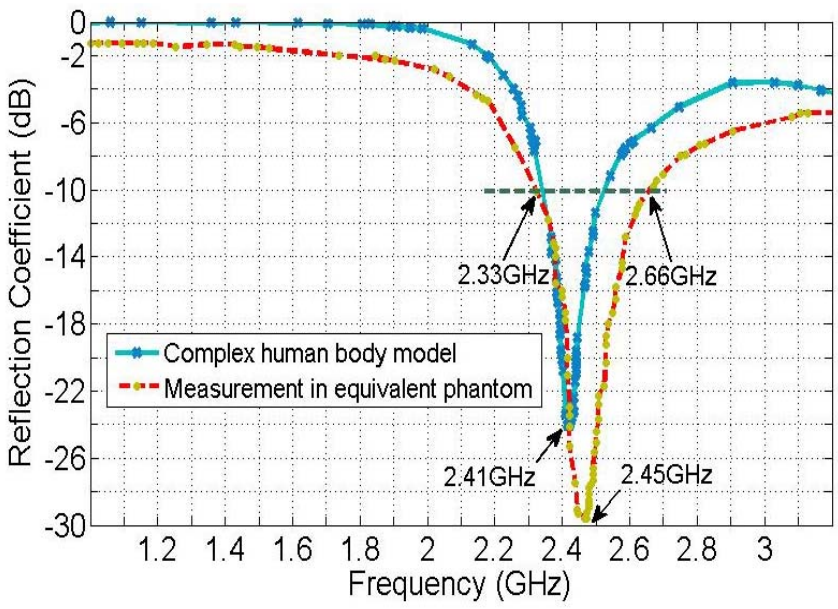

Figure 8: Reflection coefficient of Dual-slot PIFA: Measured vs. Calculated.

\section{MEASUREMENT RESULTS}

The miniaturized implantable DS-PIFA antenna prototype and the measurement set up are shown in figure 7 . The calculated result on reflection coefficient using complex human body numerical phantom is compared in figure 8 with the measured result when the antenna (including superstrate) was immersed in the mixed-meat phantom. Figure 7 reveals good agreement between the calculated and measured results on the impedance bandwidth.

We have also measured the specific absorption rate (SAR) using an indirect thermal method [8]. SAR refers to the rate at which the human body absorbs electromagnetic energy, an essential parameter to measure the human body safety under the electromagnetic wave exposure. Generally, the electric field probe scanning technique is used as a practical method for SAR measurement. However, this measurement technique requires expensive equipment. 
Table 1. Effects of variation of fat thickness and dielectric properties

\begin{tabular}{|c|c|c|c|c|}
\hline variations & \multicolumn{2}{|c|}{$\begin{array}{c}\text { Fat thickness } \\
(\mathbf{m m})\end{array}$} & \multicolumn{2}{c|}{ Fat $\mathbf{~ r}$} \\
\hline Value & $\mathbf{3}$ & $\mathbf{5}$ & $\mathbf{3 . 9}$ & $\mathbf{6 . 5}$ \\
\hline Efficiency\% & 2.3 & 2.3 & 2.1 & 2.4 \\
\hline Bandwidth $(\mathrm{MHZ})$ & 260 & 260 & 280 & 270 \\
\hline$\Delta \mathrm{f}(\mathrm{MHz})$ & -10 & 20 & -30 & 60 \\
\hline$\Delta \mathrm{f}=(\mathrm{f}-\mathrm{fr})(\mathrm{MHz}), \mathrm{fr}=2.45 \mathrm{GHz}$
\end{tabular}

Table 2. Effects of variation of skin thickness and dielectric properties.

\begin{tabular}{|c|c|c|c|c|}
\hline variations & \multicolumn{2}{|c|}{$\begin{array}{c}\text { Skin thickness } \\
(\mathbf{m m})\end{array}$} & \multicolumn{2}{c|}{ Skin $\mathbf{8 r}$} \\
\hline Value & $\mathbf{1 . 5}$ & $\mathbf{2 . 5}$ & $\mathbf{3 3}$ & $\mathbf{5 3}$ \\
\hline Efficiency\% & 2.5 & 2.1 & 2 & 2.5 \\
\hline Bandwidth $(\mathrm{MHZ})$ & 230 & 290 & 260 & 280 \\
\hline$\Delta \mathrm{f}(\mathrm{MHz})$ & -30 & 40 & -30 & 80 \\
\hline
\end{tabular}

$\Delta \mathrm{f}=(\mathrm{f}-\mathrm{fr})(\mathrm{MHz}), \mathrm{fr}=2.45 \mathrm{GHz}$

Here, we used an indirect thermal method for implantable devices when the antenna is placed inside the tissue mimicking phantom. This method offers a simple procedure for measuring the temperature rise in an equivalent human tissue caused by electromagnetic energy absorption radiated by implanted antenna. To avoid field perturbation, we have employed optical fiber thermal sensors. We measured the temperature rise in the human tissue mimicking phantom at multiple locations to evaluate the average SAR [8]. From the average temperature rise, we can calculate SAR using bio-heat transfer equation knowing tissue thermal properties which are available online. To conduct SAR measurement we have employed the same mixed meat phantom that was used to test the antenna performance. Optical thermal sensors are arranged in a 3-D arrangement to measure temperature rise $\Delta \mathrm{T}$ in the phantom which is necessary to determine the average SAR. The thermal sensors are located at 18 different measurement locations with $5 \mathrm{~mm}$ intervals over the $\mathrm{X}-\mathrm{Y}$ plane parallel with radiator surface but at two different depths $(0.5 \mathrm{~mm}$ and $1.5 \mathrm{~mm}$ ) under the skin. To measure temperature raise in phantom due to irradiation, we have used Luxtron FOT Lab Kit and optical thermal sensors. The calculated 1-g SAR for the input power level of $10 \mathrm{dBm}$ would be $5.2 \mathrm{w} / \mathrm{kg}$ while the measured result indicates $4.8 \mathrm{w} / \mathrm{kg}$ for 1 -g SAR with the same input power level. As the 1-g SAR with this level of power exceeds the FCC and European standards [11], the maximum input power must be set to $3 \mathrm{dBm}(2 \mathrm{~mW})$ to satisfy 1 -g SAR regulation.

We have also considered the effects of tissue environment variations in the calculated performance of the proposed antenna. This would be beneficial for implantation in realistic human patients as we can gain more insights about the antenna performance in such realistic situations. We anticipated the contributing factors to be the variation of dielectric properties and thickness of the fat and skin. Consequently, we varied the thickness of fat and skin layers and the dielectric properties of those tissue layers in our numerical multilayered block phantom. In each of these cases, while one of the parameters was varied, others were kept fixed. The results on impedance bandwidth, radiation efficiency and resonant frequency are tabulated in table 1 and 2. The results indicate that the proposed miniaturized PIFA antenna can cover the $2.45 \mathrm{GHz}$ ISM band even when the tissue parameters vary making it very suitable for realistic implantation.

\section{CONCLUSIONS}

A novel miniaturized implantable PIFA antenna resonating at $2.45 \mathrm{GHz}$ with a measured $330 \mathrm{MHz}$ bandwidth is presented for implantable biomedical applications. The antenna has a small size of $8.1 \times 7.1 \mathrm{~mm}^{2}$ and occupies a volume of $92 \mathrm{~mm}^{3}$ only, the antenna has a reasonable calculated radiation efficiency of $2.5 \%$ when the immersed in a lossy human tissue mimicking phantom. Calculations using different types of human tissue mimicking numerical phantoms were carried out and obtained good agreement with measurements made using mixed-meat phantom an indirect thermal method was used to measure SAR for the safety assessment. The effects of variation of tissue environment were also investigated.

\section{REFERENCES}

[1] A. Kiourti, K. Psathas, P. Lelovas, N. Kostomitsopoulos, and K.S. Nikita, In Vivo Tests of Implantable Antennas in Rats: Antenna Size and Intersubject Considerations, IEEE Antennas Wireless Propag. Lett, Vol. 12, pp. 1396-1399, 2013.

[2] M. Scarpello, et.al., Design of an Implantable Slot Dipole Conformal Flexible Antenna for Biomedical Applications, IEEE Trans. Antennas Propag., vol. 59, no.10, pp. 35563564, Oct. 2011.

[3] A. Rosen, et.al., Dynamic Evaluation of a Digital Wireless Intracranial Pressure Sensor for the Assessment of Traumatic Brain Injury in a Swine Model, IEEE Trans. Microw. Theory Tech., vol. 61, no. 1, pp. 316-325, Jan. 2013.

[4] F. Merli, L. Bolomey, J. Zürcher, G. Corradini, E. Meurville, and A. Skrivervik, Design, Realization and Measurements of a Miniature Antenna for Implantable Wireless Communication Systems, IEEE Trans. Antennas Propag., vol. 59, no.10, pp. 3544-3555, Oct. 2011.

[5] C. Liu, Y. Guo, and S. Xiao, Capacitively Loaded Circularly Polarized Implantable Patch Antenna for ISM Band Biomedical Applications, IEEE Trans. Antennas Propag., vol. 62, no.5, pp. 2407-2417, May 2014.

[6] D.K. Neog, S.S. Pattnaik, D.C. Panda, S. Devi, M. Dutta, and O. Bajpai, New expression for the resonance frequency of an E-shaped microstrip patch antenna, Microwave Opt Tech. Lett. 48, pp. 1561-1563, 2006.

[7] $\mathrm{FEKO}^{\mathrm{TM}}$, EM Software \& Systems, http://www.feko.info

[8] Y. Okano, and H. Shimoji, Comparison Measurement for Specific Absorption Rate With Physically Different Procedure, IEEE Trans. Instrumentation and Measure., vol. 61, no.2, pp. 439-446, Feb. 2012.

[9] K. Saito, Y. Hayashi, H. Yoshimura and K. Ito, "Heating Characteristics of Array Applicator Composed of Two Coaxial-Slot Antennas for Microwave Coagulation Therapy, IEEE Trans. Microw. Theory Tech., vol. 48, no.11, pp. 1800-1806, Nov. 2000.

[10] Dielectric Properties of Body Tissues (IFAC), Available: http://niremf.ifac.cnr.it/tissprop/

[11] IEEE standard for safety levels with respect to human exposure to radio frequency electromagnetic fields, $3 \mathrm{KHz}$ t0 $300 \mathrm{GHz}$, IEEE standard C95.1-2005 (Revision of IEEE standard C95.1-1991), 2006, pp. 0_1-238. 